Physical Sciences | Gao Liu

\section{Inspiration from surfactants gives Li-S cells longer life}

\author{
The renewable-fuelled future \\ is reliant on improvements \\ in battery energy density to \\ smooth the grid and power \\ journeys. Lithium-ion cells - the \\ industry standard - are almost \\ at their capacity. Lithium-sulfur \\ cells could be the answer, \\ current technology degrades \\ quickly due to an effect called \\ redox shuttling. Dr Gao Liu, \\ a staff scientist and his team \\ at the Lawrence Berkeley \\ intiration from surf have taken \\ to redesign electrolytes. \\ Hydrofluoroether (HFE) \\ additives encapsulate lithium \\ ions in micelle-like structures \\ without problematic polysulfides \\ dissolution.

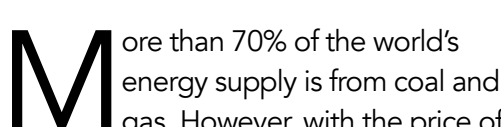 \\ II-ION CELLS: UP TO THE JOB? \\ gas. However, with the price of \\ technology is oq renewables, new \\ so09 and 2019 - thr hped $8 \%$ betwe \\ Solar only recently the most expensive \\ merawatt-hours on the market is now \\ cheaper than gas, nuclear and coal. \\ However, renewable energy in the \\ U.S. still stood at a rather meagre \\ $10 \%$ of all energy consumption in \\ 2019. So what stands in the way of the \\ renewable revolution? \\ One obvious problem is the availability \\ of these megawatt-hours. Solar panels \\ can only output electricity when the sun is \\ shining, and turbines only wum when the \\ wind is blowing. \\ The other big barrier is the transportation \\ of this power. Vehicles are a large energy

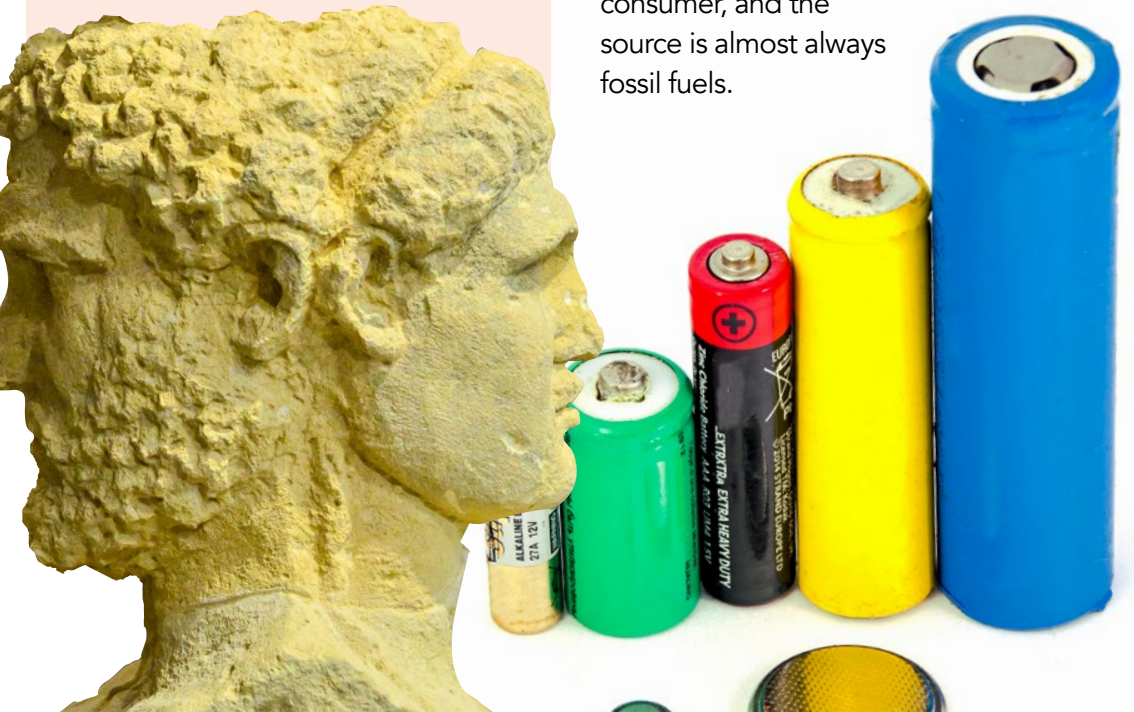 \\ energy from intermittent renewable \\ sources. Since lithium-ion cells first hit \\ the market in 2019, they have received \\ enormous investment. Battery packs \\ cost $11 \%$ of what they did a decade ago, \\ and are used for industrial electricity \\ storage for grid-balancing down to the \\ mobile phones. \\ hat there's a hard limit on the energy \\ density of lithium-ion cells, in the region of \\ 400 Watt-hours per kilogram. \\ Without improving energy density, \\ batteries remain bulky, electric vehicles \\ remain heavy, and the grid will remain \\ the 400 Wh/kg limitres. Beaching \\ batery technology at a chemical level. \\ LI-S: THE REDOX SHUTTLE \\ One exciting emerging technology is \\ higher theoretical energy density - a \\ $2600 \mathrm{Wh} / \mathrm{kg}$ - using abundant and \\ nexpensive materials. \\ A Li-S cell contains two electrodes: the \\ node composed of lithium metal an \\ the cathode composed of sulfur. As \\ it discharges, lithium dissolves in the \\ electrolyte between the electrodes and \\ travels to the cathode. At the cathode, $S_{e}$ \\ ulfur molecules react with lithium ions $L$ \\ forming $\mathrm{Li}_{2} \mathrm{~S}_{8}$ \\ As more lithium travels to the cathod
}

( further reactions occur, forming other
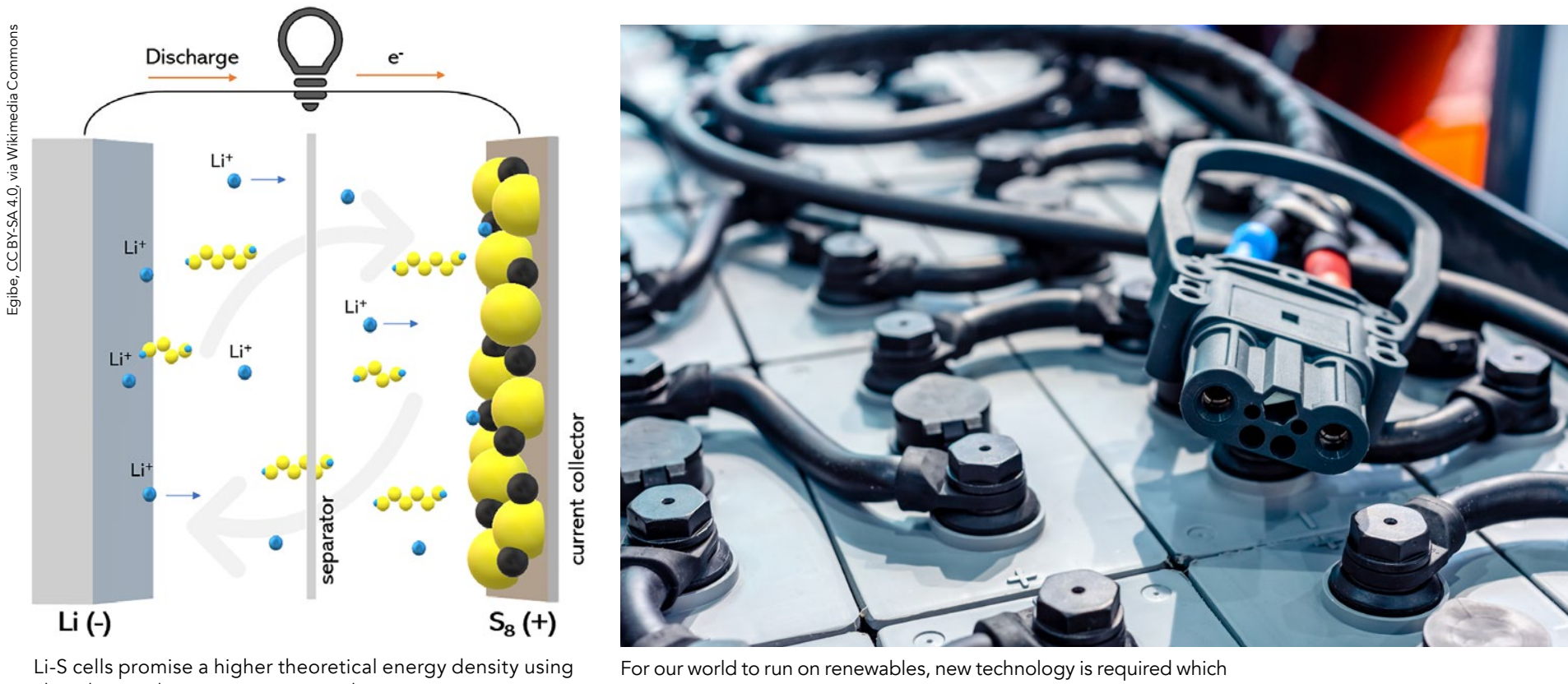

abundant and inexpensive materials

a

lithium polysulfide molecules: $\mathrm{Li}_{2} \mathrm{~S}_{\mathrm{i}_{6}}$, then

$L_{2} S_{4} S_{1} L_{2} S_{2}$, and eventually $L_{i} S$. Durng

charging, the opposite occurs.

Or at least, that's the theory. Lithium

polysulfide $\mathrm{L}_{2} \mathrm{~S}_{8}$ and $\mathrm{L}_{2} \mathrm{~S}_{6}$ are highly

soluble in the electrolyte of the cell. As

When a lithium polysulfide molecule

reaches the lithium anode, it reacts to

form a shorter-chain

polysulfide like $\mathrm{L}_{2} \mathrm{~S}_{4}$

and $\mathrm{L}_{2} \mathrm{~S}_{2,}$, depositing

some sulfur at the

slow lithium diffusion leads to poor

shuttling. Instead of modifying the electrodes, their work involves modifying the electrolyte which is sandwich

cell performance.

This approach has some heritage in the research literature: other groups have found certain electrolytes which have a

INSPIRATION FROM SURFACTANTS The Liu group have recently published a work detailing how they have taken electrolytes with "Iocalised high concentrations" of lithium salts. Their work involves using

anode.

(MFEs), a class of solvents that has

This molecule can

travel between

fortoms from the sulfur lithium electrodepositing them at the

discharge cycles, this 'redox shuttling'

effect decreases the capacity of the cell.

FIXING THE REDOX SHUTTLE

Attempts to limit redox shuttling in Li-S

lution in Li-S electrolytes is a means

previously been

to a very specific end: a viable lithium-sulfur cell. salts over lithium polysulfides. HFE

have 'used up' the solvation coordination sites, there is little room for the solvation of lithium polysulfide molecules.

However, the conclusion of much of this research is a resort to high-viscosity own: poor lith have a problem of their

cells have generally involved creating nt lithium polysulfide molecules from reaching the anode. . example, by modlying the lithium electrode to only allow lithium ions through - is complicated and costly

Dr Gao Liu is a staff scientist at the Lawrence Berkeley National Laboratory. different approach to limiting a

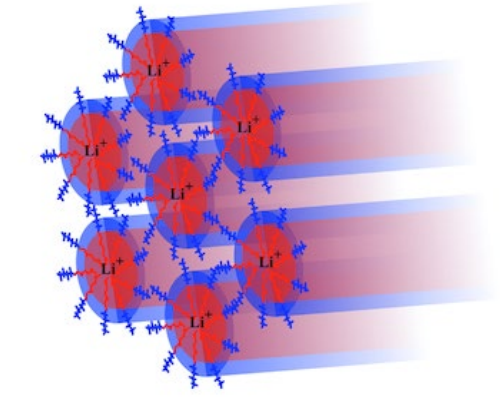

until now have generally contained a central oxygen atom, with fluorinated carbon chains on either side. The oxygen atom binds to lithium, whereas the fluorinated chains are chemically iner.

These fluorinated chains are the reason for the solvation preference of lithium ons over lithium polysulfides. The fluorinated chains block the approach of a bulky lithium polysulfide molecule to the oxygen atom. However, small lithium ions are able to approach the oxygen atom unhindered.

The Liu group's most recent research takes this idea one step further, instead central position of the solvent a they have taken inspiration from 
surfactants, to use HFEs with an ether

These molecules are designed to mic surfactant molecules, which have allowing the dissolution of oil molecules in water. In the same vein, the ether section of these specialised HFE molecules is considered 'lithiophilic' (lithium-loving) and the fluorinated section 'lithiophobic'. Liu refers to this as a "Janus" structure, in eference to the two-faced Roman god.

\section{CAN THESE FORM MICELLES?} The researchers hypothesise that when this HFE is added to a fluorinated solvent lithium salts are solvated in a special mechanism, involving the formation of structures are a shell of HFE molecules, ithiophilic ether sectionf facing inues, towards a bundle of lithium salts.

Initial tests indicate that these 'Janus' HFEs do achieve the holy grail of Li-S cells: high solubility of lithium salt with suppressed dissolution of lithium polysulfide.

These tests suggest the presence of a new solvation mechanism, proving the existence of micelle-like structures requires a specialist spectroscopic tool, called small angle $X$-ray scattering (SAXS). This is commonly used in colloid esearch to identify the size of particles oil-water suspensions

SAXS data shows that particle sizes in The of lithin salt, as well as the the of 'Janus' HFE molecule used as an additite.

The concentration of lithium salt also changed the size of the micelle-like structures, with concentrations between 0.2 and $0.3 \mathrm{~mol} \mathrm{dm}^{-3}$ leading to the largest structures, around $35 \mathrm{~nm}$ in
size. This makes intuitive sense: lower concentrations than this of lithium salts free up more HFE molecules, allowing structures On the other han higher concentrations lead to more complex structures, like lamellae - bubbles with bubbles - which would provide SAXS data that implies smaller structures are present.

This is good evidence that the micelle-like structures are spontaneously formed, and is the first report of a micelle-like structure being used in a lithium-cell electrolyte.

\section{A MEANS TO AN END}

Suppressing lithium polysulfide

dissolution in LI-S electrolytes is a mean to a very specific end: a viable lithium sufur cell. lis important to ensure that these micelle-like structures a
electrochemically stable.

\section{Key signs of success are coulombic}

outperforming it on both initial capaciy retain its capacity, eve

Crucially, the decay seen in the benchmark electrolyte - a result of its polysulfide dissolution - was largely avoided by the use of HFE additives.

The difference in capacity and coulombic efficiency between the 1:5 and 1:1 ratio electrolytes demonstrates that an excessive amount of HFE additive can prevent lithium from being transported

\section{EVIDENCE FOR SUPERIOR} PERFORMANCE A final important factor in cell life is the Affect of each charge cycle on the lithium anode. To test this, the group created test cells with one lithium electrode - as electrode with a copper one These tests were designed to establish whether the

Amphiphilic HFE molecules open up the possibility of efficient, high-capacity cells without needing to compromise on the lifetime of a product.

other words, is energy is wasted during charging, and does the cell retain its capacity after repeated charge-discharg cycles? To test these, the group measured the cycling performance of various $L i-5$ celld electrolytes composed of an HFE tetraflu in $1,1,2,2$ additive/solvent ratios. These were compared to a benchmark electrolyt DOL in DME.

Whilst a 1:1 ratio of HFE to TTE performed poorly on coulombic performed far better than the benchmark,
HFE additives are chemically stable when used alongside the lithium electrode.

These tests demonstrate that the group electrolyte is more chemically stable than charge-discharge cycles, the coulombic charge-discharge cycles, the coulombic only slightly compromised at the highes current densities tested.

Scanning electron microscope images electrodes remained smooth, indicating good chemical compatibility of the cell's chemical components.

"This technology is exciting progress cells," said Yangzhi Zhao, a postdoc researcher from Lit's group, "Amphiphil c HFE moleculas op an up the possibility of efficient, high-energy density cells witho redng to conpther on the lifuine of electric vaicles or industrialscale grid-

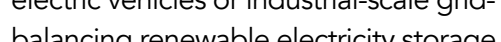

\section{Behind the Research (4) \\ Dr Yangzhi Zhao \\ $\mathrm{Dr}$ Gao Liu}

Research Objectives Dr Liu's lab combines synthetic chemistry, composite
engineering and electrochemistry to solve interdisciplinary problems in energy generation, storage and usage. The fundamental and critical issues in energy systems, as woll as synthetic techniques to develop new materials that research in battery and nenergy storage include Simaterials and electrode, electrolytes and additives, and solid-state conductors. Besides energy storage research, the team also performs materials and engineering research in building

\section{References}

Zhao Y, Fang C, Zhang G, Hubble D, Nallapaneni A, Zhu C ther Additives for Polysulfide Suppression and Li Meta Stabilization in Li-S Battery. Front
org/10.3389/fchem.2020.00484

\section{Personal Response}

\section{Are amphiphilic molecules enough to suppress lithium

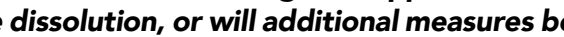

II This work has reported a proof of concept strateg amphiphilic HFE molecules as the electrolyte. The preliminary result shows effective suppression on polysulfide dissolution and shuttling as well as the additional benefit of $L$ anode stabilisation. Though promising, the current result we we could use simulation method to better understand the micelle formation and solvation mechanism, which provides useful guidance on HFE molecule design. To attempt real application, research and development in other aspects
of the Li-S cell system is simultaneously needed. This will equire the synergistic strategy by integrating optimised optimised electrode-electrolyte interfaces.

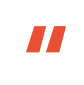

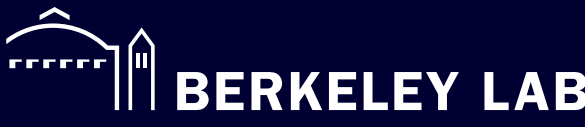

Detai

Lawrence Berkeley National Laboratory 70R0108B

1 Cyclotron Road, Berkeley, CA 94720 USA

Office Location: 070-0111

Dr Gao Liu is the Group Leader of the Applied Energy (LBNL), specialized in energy storage R\&D. Dr Liu has over 20 years of experience in developing materials and system engineering for electrical energy storage, and has led research projects for the U.S. Department of Energy and industry. Dr Liu has over 170 peer-reviewed publications and over 20 granted patent. He tis he point-of-conlact for LBN Calcharge. Dr liu also serves as board member and scientific advisor for industry.

Dr Yangzhi Zhao is a postdoc scholar in Gao Liu's lab, who is the first author on the micelle paper and currently leads the fluorinated electrolyte project. Dr Zhao specialises in materials synthesis \& manufacturing, device design, and rechargeable battery system.

This work was funded by the Assistant Secretary for

Energy Efficiency, Vehicle Technologies Office of the Materials Research (BMR) Program.

- The Advanced Light Source (beamline 7.3.3) for measurements and Molecular Foundry for NMR measurements.

-Electron Microscopy (NCEM) for electron microscopy experiments.

- All facilities are located at Lawrence Berkeley National Laboratory (LBNL), and are supported by the Director, Office of Science, Office of Basic Energy Sciences, of the 05CH11231.

Collaborators

- Particular thanks to beamline scientist Chenhui Zhu for guidance on transmission small angle X-ray (SAXS)

- The rest of researchers on this work: Yangzhi Zhao, Chen Fang, Guangzhao Zhang, Dion Hubble, Asritha Nallapane 